\title{
Lie-admissible coalgebras
}

Michel GOZE and Elisabeth REMM

Université de Haute Alsace, F.S.T., 4 rue des Frères Lumière, 68093 Mulhouse, France E-mails:m.goze@uha.fr and e.remm@uha.fr

\section{Abstract}

After introducing the concept of Lie-admissible coalgebras, we study a remarkable class corresponding to coalgebras whose coassociator satisfies invariance conditions with respect to the symmetric group $\Sigma_{3}$. We then study the convolution and tensor products.

2000 MSC: 17D25, 16W30

\section{Definitions and examples}

In this work $\mathbb{K}$ indicates a field of characteristic zero. Let $M$ be a $\mathbb{K}$-vector space and $\Delta$ a $\mathbb{K}$-linear comultiplication map $\Delta: M \rightarrow M \otimes M$. The coassociator of $\Delta$ is denoted by

$$
\tilde{A}(\Delta)=(\Delta \otimes I d) \circ \Delta-(I d \otimes \Delta) \circ \Delta
$$

and the flip $\tau: M^{\otimes 2} \rightarrow M^{\otimes 2}$ is the linear map defined by $\tau(x \otimes y)=y \otimes x$.

Let $\Sigma_{3}$ be the symmetric group of degree 3 . We denote by $c_{1}$ and $c_{2}$ the 3-cycles of $\Sigma_{3}$ and $\tau_{i j}$ the transposition echanging $i$ and $j$. For every $\sigma \in \Sigma_{3}$, we define a linear map $\Phi_{\sigma}^{M}: M^{\otimes 3} \rightarrow M^{\otimes 3}$ by

$$
\Phi_{\sigma}^{M}\left(x_{1} \otimes x_{2} \otimes x_{3}\right)=x_{\sigma^{-1}(1)} \otimes x_{\sigma^{-1}(2)} \otimes x_{\sigma^{-1}(3)}
$$

Definition 1. The pair $(M, \Delta)$ is a Lie-admissible coalgebra if the linear map $\Delta_{L}: M \rightarrow M \otimes M$ defined by $\Delta_{L}=\Delta-\tau \circ \Delta$ is a Lie coalgebra comultiplication, that is, if $\Delta_{L}$ satisfies

$$
\left\{\begin{array}{l}
\tau \circ \Delta_{L}=-\Delta_{L} \\
\tilde{A}\left(\Delta_{L}\right)+\Phi_{c_{1}}^{M} \circ \tilde{A}\left(\Delta_{L}\right)+\Phi_{c_{2}}^{M} \circ \tilde{A}\left(\Delta_{L}\right)=0
\end{array}\right.
$$

Recall that a multiplication $\mu$ of a $\mathbb{K}$-algebra $(\mathcal{A}, \mu)$ is Lie-admissible if its associator

$$
A(\mu)=\mu \circ(\mu \otimes I d)-\mu \circ(I d \otimes \mu)
$$

satisfies

$$
\Sigma_{\sigma \in \Sigma_{3}}(-1)^{\epsilon(\sigma)} A(\mu) \circ \Phi_{\sigma}^{\mathcal{A}}=0
$$

where $\epsilon(\sigma)$ is the sign of the permutation $\sigma$. This means that the algebra $(\mathcal{A},[]$,$) whose product is$ given by the bracket $[x, y]=\mu(x, y)-\mu(y, x)$ is a Lie algebra. We have a similar characterization of a Lie-admissible comultiplication.

Proposition 1. A comultiplication $\Delta$ on $M$ is a Lie-admissible comultiplication if and only if $\Delta$ satisfies

$$
\sum_{\sigma \in \Sigma_{3}}(-1)^{\epsilon(\sigma)} \Phi_{\sigma}^{M} \circ \tilde{A}(\Delta)=0
$$

where $\epsilon(\sigma)$ denotes the sign of the permutation $\sigma$.

Proof. It is a direct consequence of Equation (1.1) because

$$
\begin{aligned}
\tilde{A}(\tau \circ \Delta) & =((\tau \circ \Delta) \otimes I d) \circ \tau \circ \Delta-(I d \otimes(\tau \circ \Delta)) \circ(\tau \circ \Delta) \\
& =-\left(\Phi_{\tau_{13}}^{M} \circ \tilde{A}\right)(\Delta)
\end{aligned}
$$

This proves the proposition. 


\section{Examples}

- Every coassociative coalgebra is a Lie-admissible coalgebra.

- The comultiplication of a pre-Lie coalgebra $(M, \Delta)$ satisfies

$$
\tilde{A}(\Delta)-\Phi_{\tau_{23}}^{M} \circ \tilde{A}(\Delta)=0
$$

Since the composition of (1.3) by $\Phi_{c_{1}}^{M}$ and $\Phi_{c_{2}}^{M}$ gives respectively

$$
\Phi_{c_{1}}^{M} \circ \tilde{A}(\Delta)-\Phi_{\tau_{13}}^{M} \circ \tilde{A}(\Delta)=0
$$

and

$$
\Phi_{c 2}^{M} \circ \tilde{A}(\Delta)-\Phi_{\tau_{12}}^{M} \circ \tilde{A}(\Delta)=0
$$

we obtain Identity (1.2) by summation of (1.3) with these two equations and every pre-Lie coalgebra is Lie-admissible.

In the following sections we generalize these examples.

\section{$2 \quad G_{i}$-coalgebras}

An interesting class of Lie-admissible coalgebras is obtained by dualizing the $G_{i}$-associative algebras. These Lie-admissible algebras has been introduced in [9] and developed in [3]. Let us point out these initially notations.

\section{$2.1 \quad \Sigma_{3}$-associative algebras}

Let $\mathbb{K}\left[\Sigma_{3}\right]$ be the group algebra associated to $\Sigma_{3}$, where $\mathbb{K}$ is a field of characteristic zero. Every $v \in \mathbb{K}\left[\Sigma_{3}\right]$ decomposes as follows:

$$
v=a_{1} i d+a_{2} \tau_{12}+a_{3} \tau_{13}+a_{4} \tau_{23}+a_{5} c_{1}+a_{6} c_{2}
$$

or simply

$$
v=\sum_{\sigma \in \Sigma_{3}} a_{\sigma} \sigma
$$

where $a_{\sigma} \in \mathbb{K}$. If $\mathcal{A}$ is a $\mathbb{K}$-vector space, then we define from such a vector $v$ the endomorphism $\Phi_{v}^{\mathcal{A}}$ of $\mathcal{A}^{\otimes 3}$ by

$$
\Phi_{v}^{\mathcal{A}}=\sum_{\sigma \in \Sigma_{3}} a_{\sigma} \Phi_{\sigma}^{\mathcal{A}}
$$

Consider the natural right action of $\Sigma_{3}$ on $\mathbb{K}\left[\Sigma_{3}\right]$ :

$$
\Sigma_{3} \times \mathbb{K}\left[\Sigma_{3}\right] \rightarrow \mathbb{K}\left[\Sigma_{3}\right], \quad\left(\sigma, \sum_{i} a_{i} \sigma_{i}\right) \mapsto \sum_{i} a_{i} \sigma^{-1} \circ \sigma_{i}
$$

The corresponding orbit of a vector $v \in \mathbb{K}\left[\Sigma_{3}\right]$ is denoted by $\mathcal{O}(v)$ and generates a linear subspace $F_{v}=\mathbb{K}(\mathcal{O}(v))$ of $\mathbb{K}\left[\Sigma_{3}\right]$. It is an invariant subspace of $\mathbb{K}\left[\Sigma_{3}\right]$. Therefore, using Mashke's theorem, it is a direct product of irreducible invariant subspaces.

Let $(\mathcal{A}, \mu)$ be a $\mathbb{K}$-algebra with multiplication $\mu$ and $A(\mu)$ its associator. 
Definition 2. An algebra $(\mathcal{A}, \mu)$ is a $\Sigma_{3}$-associative algebra if there exists $v \in \mathbb{K}\left[\Sigma_{3}\right], v \neq 0$ such that $A(\mu) \circ \Phi_{v}^{\mathcal{A}}=0$.

Proposition 2. Let $v$ be in $\mathbb{K}\left[\Sigma_{3}\right]$ such that dim $F_{v}=1$. Then $v=\alpha V$ or $v=\alpha W$ with $\alpha \in \mathbb{K}$ and the vectors $V$ and $W$ are the following vectors:

$$
\begin{aligned}
V & =I d-\tau_{12}-\tau_{13}-\tau_{23}+c_{1}+c_{2} \\
W & =I d+\tau_{12}+\tau_{13}+\tau_{23}+c_{1}+c_{2}
\end{aligned}
$$

The first case corresponds to the character of $\Sigma_{3}$ given by the sign, the second corresponds to the trivial case.

Every algebra $(\mathcal{A}, \mu)$ whose associator satisfies

$$
A(\mu) \circ \Phi_{V}^{\mathcal{A}}=0
$$

is a Lie-admissible algebra. Likewise an algebra $(\mathcal{A}, \mu)$ whose associator satisfies

$$
A(\mu) \circ \Phi_{W}^{\mathcal{A}}=0
$$

is 3 -power associative, that is, it satisfies $A(\mu)(x, x, x)=0$ for every $x \in A$.

\section{$2.2 \quad G_{i}$-associative algebras}

The class of $\Sigma_{3}$-associative Lie-admissible algebras contains interesting subclasses associated to the subgroups $G_{i}$ of $\Sigma_{3}$ that we naturally call $G_{i}$-associative algebras. Let us introduce some notations. Consider the subgroups of $\Sigma_{3}$ :

$$
\begin{array}{lll}
G_{1}=\{I d\}, & G_{2}=\left\{I d, \tau_{12}\right\}, & G_{3}=\left\{I d, \tau_{23}\right\} \\
G_{4}=\left\{I d, \tau_{13}\right\}, & G_{5}=\left\{I d, c_{1}, c_{2}\right\}=A_{3}, & G_{6}=\Sigma_{3}
\end{array}
$$

Definition 3. Let $G_{i}$ be a subgroup of $\Sigma_{3}$. The algebra $(\mathcal{A}, \mu)$ is $G_{i^{-}}$associative if

$$
\sum_{\sigma \in G_{i}}(-1)^{\varepsilon(\sigma)} A(\mu) \circ \Phi_{\sigma}^{\mathcal{A}}=0
$$

Proposition 3. Every $G_{i}$-associative algebra is a $\Sigma_{3}$-associative algebra.

Proof. Every subgroup $G_{i}$ of $\Sigma_{3}$ corresponds to an invariant linear space $F\left(v_{i}\right)$ generated by a single vector $v_{i} \in \mathbb{K}\left[\Sigma_{3}\right]$. More precisely we consider $v_{1}=I d, v_{2}=I d-\tau_{12}, v_{3}=I d-\tau_{23}$, $v_{4}=I d-\tau_{13}, v_{5}=I d+c_{1}+c_{2}$ and $v_{6}=V$ that we have defined in (2.1).

Proposition 4. Every $G_{i}$-associative algebra is a Lie-admissible algebra.

Proof. The vector $V$ belongs to the orbits $\mathcal{O}\left(v_{i}\right)$ for every $v_{i}$. Thus, if $\mu$ is a $G_{i}$-associative product, it also satisfies

$$
A(\mu) \circ \Phi_{V}^{\mathcal{A}}=0
$$

and $\mu$ is a Lie-admissible multiplication.

We deduce the following type of Lie-admissible algebras:

1. A $G_{1}$-associative algebra is an associative algebra.

2. A $G_{2}$-associative algebra is a Vinberg algebra. If $A$ is finite-dimensional, the associated Lie algebra is provided with an affine structure. 
3. A $G_{3}$-associative algebra is a pre-Lie algebra.

4. If $(\mathcal{A}, \mu)$ is $G_{4}$-associative then $\mu$ satisfies

$$
(X \cdot Y) \cdot Z-X \cdot(Y \cdot Z)=(Z \cdot Y) \cdot X-Z \cdot(Y \cdot X)
$$

with $X \cdot Y=\mu(X, Y)$.

5. If $(\mathcal{A}, \mu)$ is $G_{5}$-associative then $\mu$ satisfies the generalized Jacobi condition :

$$
(X \cdot Y) \cdot Z+(Y \cdot Z) \cdot X+(Z \cdot X) \cdot Y=X \cdot(Y \cdot Z)+Y \cdot(Z \cdot X)+Z \cdot(X \cdot Y)
$$

with $X \cdot Y=\mu(X, Y)$. Moreover if the product is skew-symmetric, then it is a Lie algebra bracket.

6. A $G_{6}$-associative algebra is a Lie-admissible algebra.

\section{$2.3 \quad G_{i}$-coalgebras}

Dualizing the formula $(2.3)$ we obtain the notion of $G_{i}$-coalgebra.

Definition 4. A $G_{i}$-coalgebra is a $\mathbb{K}$-vector space $M$ provided with a comultiplication $\Delta$ satisfying

$$
\sum_{\sigma \in G_{i}}(-1)^{\epsilon(\sigma)} \Phi_{\sigma}^{M} \circ \tilde{A}(\Delta)=0
$$

Remark. We can present an equivalent and axiomatic definition of the notion of $G_{i}$-associative algebra. A $G_{i}$-associative algebra is $\left(\mathcal{A}, \mu, \eta, G_{i}\right)$ where $\mathcal{A}$ is a vector space, $G_{i}$ a subgroup of $\Sigma_{3}, \mu: \mathcal{A} \otimes \mathcal{A} \longrightarrow \mathcal{A}$ and $\eta: \mathbb{K} \rightarrow \mathcal{A}$ are linear maps satisfying the following axioms:

1. $\left(G_{i}\right.$-ass): The square

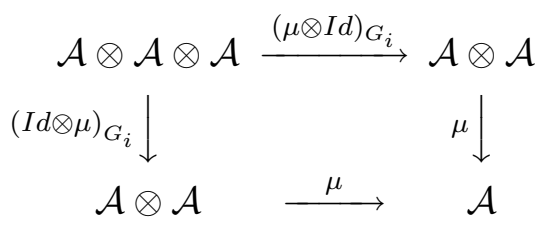

commutes, where $(I d \otimes \mu)_{G_{i}}$ is the linear mapping defined by:

$$
(I d \otimes \mu)_{G_{i}}=\sum_{\sigma \in G_{i}}(-1)^{\epsilon(\sigma)}(I d \otimes \mu) \circ \Phi_{\sigma}^{\mathcal{A}}
$$

If we impose that the algebra is unitary we have to add the following axiom:

2. (Un) The following diagram is commutative:

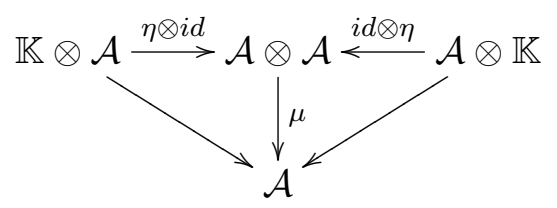

The axiom $\left(G_{i}\right.$-ass $)$ expresses that the multiplication $\mu$ is $G_{i}$-associative whereas the axiom (Un) means that the element $\eta(1)$ of $\mathcal{A}$ is a left and right unit for $\mu$. We want to dualize the previous diagrams to obtain the notions of corresponding coalgebras. Let $\Delta$ be a comultiplication $\Delta: M \longrightarrow M \otimes M$. We define the bilinear map

$$
G_{i} \circ(\Delta \otimes I d): M^{\otimes 3} \longrightarrow M^{\otimes 3}
$$


by

$$
G_{i} \circ(\Delta \otimes I d)=\sum_{\sigma \in G_{i}}(-1)^{\epsilon(\sigma)} \Phi_{\sigma}^{\mathcal{A}} \circ(\Delta \otimes I d)
$$

A $G_{i}$-coalgebra is a vector space $M$ provided with a comultiplication $\Delta: M \longrightarrow M \otimes M$ and a counit $\epsilon: M \rightarrow \mathbb{K}$ such that

1. $\left(G_{i}\right.$-ass co $)$ The following square is commutative:

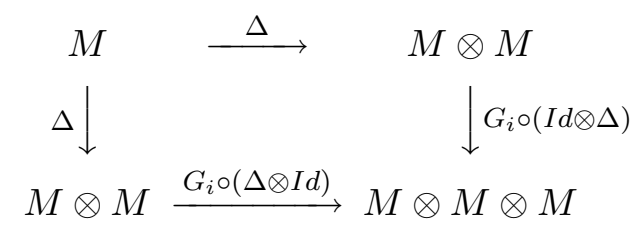

If we suppose moreover that the coalgebra is counitary we have to add the following axiom:

2. (Coun) The following diagram is commutative:

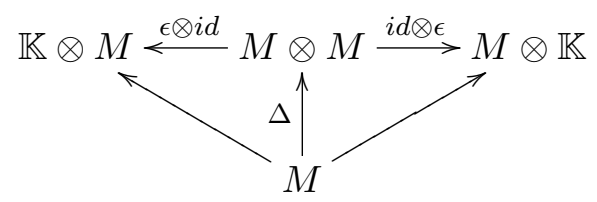

A morphism of $G_{i}$-coalgebras

$$
f:\left(M, \Delta, \epsilon, G_{i}\right) \rightarrow\left(M^{\prime}, \Delta^{\prime}, \epsilon^{\prime}, G_{i}\right)
$$

is a linear map from $M$ to $M^{\prime}$ such that

$$
(f \otimes f) \circ \Delta=\Delta^{\prime} \circ f \quad \text { and } \quad \epsilon=\epsilon^{\prime} \circ f
$$

Proposition 5. Every $G_{i}$-coalgebra is a Lie-admissible coalgebra.

Proof. The Lie-admissible coalgebras are given by the relation

$$
\sum_{\sigma \in \Sigma_{3}}(-1)^{\epsilon(\sigma)} \Phi_{\sigma}^{M} \circ \tilde{A}(\Delta)=\Phi_{V}^{M} \circ \tilde{A}(\Delta)=0
$$

Since for every $v_{i}=\sum_{\sigma \in G_{i}}(-1)^{\epsilon(\sigma)} \sigma$ we have $V \in F_{v_{i}}$, we deduce the proposition.

\subsection{The dual space of a $G_{i}$-coalgebra}

For any natural number $n$ and any $\mathbb{K}$-vector spaces $E$ and $F$, we denote by

$$
\lambda_{n}: \operatorname{Hom}(E, F)^{\otimes n} \longrightarrow \operatorname{Hom}\left(E^{\otimes n}, F^{\otimes n}\right)
$$

the natural embedding

$$
\lambda_{n}\left(f_{1} \otimes \ldots \otimes f_{n}\right)\left(x_{1} \otimes \ldots \otimes x_{n}\right)=f_{1}\left(x_{1}\right) \otimes \ldots \otimes f_{n}\left(x_{n}\right)
$$

Proposition 6. The dual space of a $G_{i}$-coalgebra is provided with a structure of $G_{i}$-associative algebra. 
Proof. Let $(M, \Delta)$ be a $G_{i}$-coalgebra. We consider the multiplication on the dual vector space $M^{*}$ of $M$ defined by $\mu=\Delta^{*} \circ \lambda_{2}$. It provides $M^{*}$ with a $G_{i}$-associative algebra structure. In fact we have

$$
\mu\left(f_{1} \otimes f_{2}\right)=\mu_{\mathbb{K}} \circ \lambda_{2}\left(f_{1} \otimes f_{2}\right) \circ \Delta
$$

for all $f_{1}, f_{2} \in M^{*}$ where $\mu_{\mathbb{K}}$ is the multiplication in $\mathbb{K}$. Equation (2.4) becomes:

$$
\begin{aligned}
\mu \circ(\mu \otimes I d)\left(f_{1} \otimes f_{2} \otimes f_{3}\right) & =\mu_{\mathbb{K}} \circ\left(\lambda_{2}\left(\mu\left(f_{1} \otimes f_{2}\right) \otimes f_{3}\right)\right) \circ \Delta \\
& =\mu_{\mathbb{K}} \circ \lambda_{2}\left(\left(\mu_{\mathbb{K}} \circ \lambda_{2}\left(f_{1} \otimes f_{2}\right) \circ \Delta\right) \otimes f_{3}\right) \circ \Delta \\
& =\mu_{\mathbb{K}} \circ\left(\mu_{\mathbb{K}} \otimes I d\right) \circ \lambda_{3}\left(f_{1} \otimes f_{2} \otimes f_{3}\right) \circ(\Delta \otimes I d) \circ \Delta
\end{aligned}
$$

The associator $A(\mu)$ satisfies

$$
\begin{aligned}
A(\mu)= & +\mu_{\mathbb{K}} \circ\left(\mu_{\mathbb{K}} \otimes I d\right) \circ \lambda_{3}\left(f_{1} \otimes f_{2} \otimes f_{3}\right) \circ(\Delta \otimes I d) \circ \Delta \\
& -\mu_{\mathbb{K}} \circ\left(I d \otimes \mu_{\mathbb{K}}\right) \circ \lambda_{3}\left(f_{1} \otimes f_{2} \otimes f_{3}\right) \circ(I d \otimes \Delta) \circ \Delta
\end{aligned}
$$

and using associativity and commutativity of the multiplication in $\mathbb{K}$, we obtain

$$
A(\mu)=\mu_{\mathbb{K}} \circ\left(\mu_{\mathbb{K}} \otimes I d\right) \circ \lambda_{3}\left(f_{1} \otimes f_{2} \otimes f_{3}\right) \circ((\Delta \otimes I d) \circ \Delta-(I d \otimes \Delta) \circ \Delta)
$$

Thus

$$
\begin{aligned}
\sum_{\sigma \in G_{i}}(-1)^{\epsilon(\sigma)} A(\mu) \circ \Phi_{\sigma}^{M^{*}}= & \mu_{\mathbb{K}} \circ\left(\mu_{\mathbb{K}} \otimes I d\right) \circ \lambda_{3}\left(f_{1} \otimes f_{2} \otimes f_{3}\right) \circ\left(G_{i} \circ(\Delta \otimes I d) \circ \Delta\right. \\
& \left.-G_{i} \circ(I d \otimes \Delta) \circ \Delta\right) \\
= & 0
\end{aligned}
$$

and $\left(M^{*}, \mu\right)$ is a $G_{i}$-associative algebra.

Proposition 7. The dual vector space of a finite dimensional $G_{i}$-associative algebra has a $G_{i^{-}}$ coalgebra structure.

Proof. Let $\mathcal{A}$ be a finite dimensional $G_{i}$-associative algebra and let $\left\{e_{i}, i=1, \ldots, n\right\}$ be a basis of $\mathcal{A}$. If $\left\{f_{i}\right\}$ is the dual basis then $\left\{f_{i} \otimes f_{j}\right\}$ is a basis of $\mathcal{A}^{*} \otimes \mathcal{A}^{*}$. The coproduct $\Delta$ on $\mathcal{A}^{*}$ is defined by

$$
\Delta(f)=\sum_{i, j} f\left(\mu\left(e_{i} \otimes e_{j}\right)\right) f_{i} \otimes f_{j}
$$

In particular

$$
\Delta\left(f_{k}\right)=\sum_{i, j} C_{i j}^{k} f_{i} \otimes f_{j}
$$

where $C_{i j}^{k}$ are the structure constants of $\mu$ related to the basis $\left\{e_{i}\right\}$. Then $\Delta$ is the comultiplication of a $G_{i}$-associative coalgebra.

\section{The convolution product}

Let us recall that if $(\mathcal{A}, \mu)$ is associative $\mathbb{K}$-algebra and $(M, \Delta)$ a coassociative $\mathbb{K}$-coalgebra (i.e. a $G_{1}$-coalgebra) then the convolution product

$$
f \star g=\mu \circ \lambda_{2}(f \otimes g) \circ \Delta
$$

provides $\operatorname{Hom}(M, \mathcal{A})$ with an associative algebra structure. This result can be extended to the $G_{i}$-associative algebras and coalgebras. But we have to introduce the notion of $G_{i}^{!}$-coalgebras defined by the Koszul duality in the operads theory [2] and [3]. 


\subsection{The $G_{i}^{!}$-algebras and coalgebras}

Let $G_{i}-\mathcal{A} s s$ be the quadratic operad associated to the $G_{i}$-associative algebras. In [3] and [8], we show that these operads satisfy the Koszul duality as soon as $i=1,2,3,6$. Let $G_{i}-\mathcal{A} s s^{!}$be the dual operad. We will call a $G_{i}^{!}$-algebra any algebra on $G_{i}-\mathcal{A} s s^{!}$. These algebras are defined as follows:

Definition 5. For $i \geq 2$, a $G_{i}^{!}$-algebra is an associative algebra satisfying

- for $i=2: x_{1} \cdot x_{2} \cdot x_{3}=x_{2} \cdot x_{1} \cdot x_{3}$,

- for $i=3: x_{1} \cdot x_{2} \cdot x_{3}=x_{1} \cdot x_{3} \cdot x_{2}$,

- for $i=4: x_{1} \cdot x_{2} \cdot x_{3}=x_{3} \cdot x_{2} \cdot x_{1}$,

- for $i=5: x_{1} \cdot x_{2} \cdot x_{3}=x_{2} \cdot x_{3} \cdot x_{1}=x_{3} \cdot x_{1} \cdot x_{2}$,

- for $i=6: x_{1} \cdot x_{2} \cdot x_{3}=x_{\sigma(1)} \cdot x_{\sigma(2)} \cdot x_{\sigma(3)}$ for all $x_{1}, x_{2}, x_{3}$ and $\sigma \in \Sigma_{3}$.

Definition 6. For $i \geq 2$, a $G_{i}^{!}$-coalgebra is a coassociative coalgebra satisfying $\Phi_{\sigma}^{M} \circ(I d \otimes \Delta) \circ \Delta=(I d \otimes \Delta) \circ \Delta \quad$ for every $\sigma \in G_{i}$

We will provide $\operatorname{Hom}(M, \mathcal{A})$ with a structure of $G_{i}$-associative algebra.

Proposition 8. Let $(\mathcal{A}, \mu)$ be a $G_{i}$-associative algebra and $(M, \Delta)$ a $G_{i}^{!}$-coalgebra. Then the algebra $(\operatorname{Hom}(M, \mathcal{A}), \star)$ is a $G_{i}$-associative algebra where $\star$ is the convolution product

$$
f \star g=\mu \circ \lambda_{2}(f \otimes g) \circ \Delta
$$

Proof. Let us compute the associator $A(\star)$ of the convolution product. Since

$$
\begin{aligned}
\left(f_{1} \star f_{2}\right) \star f_{3} & =\mu \circ \lambda_{2}\left(\left(f_{1} \star f_{2}\right) \otimes f_{3}\right) \circ \Delta \\
& =\mu \circ \lambda_{2}\left(\left(\mu \circ \lambda_{2}\left(f_{1} \otimes f_{2}\right) \circ \Delta\right) \otimes f_{3}\right) \circ \Delta \\
& =\mu \circ(\mu \otimes I d) \circ \lambda_{3}\left(f_{1} \otimes f_{2} \otimes f_{3}\right) \circ(\Delta \otimes I d) \circ \Delta
\end{aligned}
$$

we have

$$
\begin{aligned}
A(\star)\left(f_{1} \otimes f_{2} \otimes f_{3}\right) & =\mu \circ(\mu \otimes I d) \circ \lambda_{3}\left(f_{1} \otimes f_{2} \otimes f_{3}\right) \circ(\Delta \otimes I d) \circ \Delta \\
& -\mu \circ(I d \otimes \mu) \circ \lambda_{3}\left(f_{1} \otimes f_{2} \otimes f_{3}\right) \circ(I d \otimes \Delta) \circ \Delta
\end{aligned}
$$

Therefore

$$
\begin{aligned}
& \sum_{\sigma \in G_{i}}(-1)^{\epsilon(\sigma)} A(\star) \circ \Phi_{\sigma}^{H o m}(M, A)\left(f_{1} \otimes f_{2} \otimes f_{3}\right) \\
& =\mu \circ(\mu \otimes I d) \circ\left(\sum_{\sigma \in G_{i}} \lambda_{3}\left(\Phi_{\sigma}^{H o m}(M, A)\left(f_{1} \otimes f_{2} \otimes f_{3}\right)\right)\right) \circ(\Delta \otimes I d) \circ \Delta \\
& -\mu \circ(I d \otimes \mu) \circ\left(\sum_{\sigma \in G_{i}} \lambda_{3}\left(\Phi_{\sigma}^{H o m}(M, A)\left(f_{1} \otimes f_{2} \otimes f_{3}\right)\right)\right) \circ(I d \otimes \Delta) \circ \Delta
\end{aligned}
$$

But

$$
\lambda_{3}\left(\Phi_{\sigma}^{\operatorname{Hom}(M, A)}\left(f_{1} \otimes f_{2} \otimes f_{3}\right)\right)=\Phi_{\sigma}^{A} \circ \lambda_{3}\left(f_{1} \otimes f_{2} \otimes f_{3}\right) \circ \Phi_{\sigma^{-1}}^{M}
$$

This gives

$$
\begin{aligned}
& \sum_{\sigma \in G_{i}}(-1)^{\epsilon(\sigma)} A(\star) \circ \Phi_{\sigma}^{H o m}(M, A)\left(f_{1} \otimes f_{2} \otimes f_{3}\right) \\
& =\mu \circ(\mu \otimes I d) \circ\left(\sum_{\sigma \in G_{i}} \Phi_{\sigma}^{A} \circ \lambda_{3}\left(f_{1} \otimes f_{2} \otimes f_{3}\right)\right) \circ \Phi_{\sigma^{-1}}^{M} \circ(\Delta \otimes I d) \circ \Delta \\
& -\mu \circ(I d \otimes \mu) \circ\left(\sum_{\sigma \in G_{i}} \Phi_{\sigma}^{A} \circ \lambda_{3}\left(f_{1} \otimes f_{2} \otimes f_{3}\right)\right) \circ \Phi_{\sigma^{-1}}^{M} \circ(I d \otimes \Delta) \circ \Delta
\end{aligned}
$$


Since $\Delta$ is coassociative,

$$
(\Delta \otimes I d) \circ \Delta=(I d \otimes \Delta) \circ \Delta
$$

the $G_{i}^{!}$-coalgebra structure implies

$$
\Phi_{\sigma}^{M} \circ(I d \otimes \Delta) \circ \Delta=\Phi_{\sigma}^{M} \circ(\Delta \otimes I d) \circ \Delta=(\Delta \otimes I d) \circ \Delta
$$

Then

$$
\begin{aligned}
& \sum_{\sigma \in G_{i}}(-1)^{\epsilon(\sigma)} A(\star) \circ \Phi_{\sigma}^{H o m}(M, A)\left(f_{1} \otimes f_{2} \otimes f_{3}\right) \\
& =\mu \circ(\mu \otimes I d) \circ\left(\sum_{\sigma \in G_{i}} \Phi_{\sigma}^{A} \circ \lambda_{3}\left(f_{1} \otimes f_{2} \otimes f_{3}\right)\right) \circ(\Delta \otimes I d) \circ \Delta \\
& -\mu \circ(I d \otimes \mu) \circ\left(\sum_{\sigma \in G_{i}} \Phi_{\sigma}^{A} \circ \lambda_{3}\left(f_{1} \otimes f_{2} \otimes f_{3}\right)\right) \circ(\Delta \otimes I d) \circ \Delta \\
& =\sum_{\sigma \in G_{i}} A(\mu) \circ \Phi_{\sigma}^{A} \circ \lambda_{3}\left(f_{1} \otimes f_{2} \otimes f_{3}\right) \circ(\Delta \otimes I d) \circ \Delta \\
& =0
\end{aligned}
$$

This proves the proposition.

\subsection{Lie-admissible bialgebras.}

Definition 7. A Lie-admissible bialgebra is a triple $(\mathcal{A}, \mu, \Delta)$ where $(\mathcal{A}, \mu)$ is a Lie-admissible algebra and $(\mathcal{A}, \Delta)$ a Lie-admissible coalgebra with a compatibility condition between $\Delta$ and $\mu$ :

$$
\Delta \circ A(\mu) \circ \Phi_{G_{6}}^{\mathcal{A}}=0
$$

Here we do not assume that the algebra and coalgebra are unitary and counitary. Among Lieadmissible bialgebras, we shall have the class of $G_{i}$-bialgebras. As example, a compatibility condition for pre-Lie bialgebras (that is $G_{3}$-bialgebras) is given by

$$
\Delta \circ \mu=(I d \otimes \mu) \circ(\Delta \otimes I d)+(\mu \otimes I d) \circ \Phi_{\tau_{23}}^{\mathcal{A}} \circ(\Delta \otimes I d)
$$

\section{Tensor product of Lie-admissible algebras and coalgebras}

\subsection{Tensor product of $G_{i}$ and $G_{i}^{!}$-algebras}

We know that the tensor product of associative algebras can be provided with an associative algebra structure. In other words, the category of associative algebras is monoidal and closed for the tensor product. This is not true in general for other categories of $\Sigma_{3}$-associative algebras.

Proposition 9. Let $\left(\mathcal{A}, \mu_{A}\right)$ and $\left(\mathcal{B}, \mu_{B}\right)$ be two $\Sigma_{3}$-associative algebras respectively defined by the relations $A\left(\mu_{A}\right) \circ \Phi_{v}^{\mathcal{A}}=0$ and $A\left(\mu_{B}\right) \circ \Phi_{w}^{\mathcal{B}}=0$. Then $\left(\mathcal{A} \otimes_{\mathbb{K}} \mathcal{B}, \mu_{A} \otimes \mu_{B}\right)$ is a $\Sigma_{3}$-associative algebra if and only if $\mathcal{A}$ and $\mathcal{B}$ are associative algebras (i.e $G_{1}$-associative algebras).

Proof. See [4].

But we have:

Theorem 10. If $\mathcal{A}$ is a $G_{i}$-associative algebra and $\mathcal{B}$ a $G_{i}^{!}$-algebra (with the same index) then $\mathcal{A} \otimes \mathcal{B}$ can be provided with a $G_{i}$-algebra structure for $i=1, \ldots, 6$. 
Proof. Let us consider on $\mathcal{A} \otimes \mathcal{B}$ the classical tensor product

$$
\mu_{A} \otimes \mu_{B}\left(\left(a_{1} \otimes b_{1}\right) \otimes\left(a_{2} \otimes b_{2}\right)\right)=\mu_{A}\left(a_{1} \otimes a_{2}\right) \otimes \mu_{B}\left(b_{1} \otimes b_{2}\right)
$$

To simplify, we denote by $\mu$ the product $\mu_{A} \otimes \mu_{B}$. As $\mathcal{B}$ is an associative algebra, the associator $A(\mu)$ satisfies

$$
A(\mu)\left(\left(a_{1} \otimes b_{1}\right) \otimes\left(a_{2} \otimes b_{2}\right) \otimes\left(a_{3} \otimes b_{3}\right)\right)=A\left(\mu_{A}\right)\left(a_{1} \otimes a_{2} \otimes a_{3}\right) \otimes \mu_{B} \circ\left(\mu_{B} \otimes I d\right)\left(b_{1} \otimes b_{2} \otimes b_{3}\right)
$$

Therefore

$$
\begin{aligned}
\sum_{\sigma \in G_{i}} & (-1)^{\epsilon(\sigma)} A(\mu) \circ \Phi_{\sigma}^{\mathcal{A} \otimes \mathcal{B}}\left(\left(a_{1} \otimes b_{1}\right) \otimes\left(a_{2} \otimes b_{2}\right) \otimes\left(a_{3} \otimes b_{3}\right)\right) \\
& =\sum_{\sigma \in G_{i}}(-1)^{\epsilon(\sigma)} A\left(\mu_{A}\right) \circ \Phi_{\sigma}^{\mathcal{A}}\left(a_{1} \otimes a_{2} \otimes a_{3}\right) \otimes \mu_{B} \circ\left(\mu_{B} \otimes I d\right) \circ \Phi_{\sigma}^{\mathcal{B}}\left(b_{1} \otimes b_{2} \otimes b_{3}\right)
\end{aligned}
$$

But $\mathcal{B}$ a $G_{i}^{!}$-algebra. Then

$$
\mu_{B} \circ\left(\mu_{B} \otimes I d\right) \circ \Phi_{\sigma}^{\mathcal{B}}\left(b_{1} \otimes b_{2} \otimes b_{3}\right)=\mu_{B} \circ\left(\mu_{B} \otimes I d\right)\left(b_{1} \otimes b_{2} \otimes b_{3}\right)
$$

for any $\sigma \in G_{i}$. So we obtain

$$
\begin{aligned}
\sum_{\sigma \in G_{i}}( & -1)^{\epsilon(\sigma)} A(\mu) \circ \Phi_{\sigma}^{\mathcal{A} \otimes \mathcal{B}}\left(\left(a_{1} \otimes b_{1}\right) \otimes\left(a_{2} \otimes b_{2}\right) \otimes\left(a_{3} \otimes b_{3}\right)\right) \\
& =\left(\sum_{\sigma \in G_{i}}(-1)^{\epsilon(\sigma)} A\left(\mu_{A}\right) \circ \Phi_{\sigma}^{\mathcal{A}}\left(a_{1} \otimes a_{2} \otimes a_{3}\right)\right) \otimes \mu_{B} \circ\left(\mu_{B} \otimes I d\right)\left(b_{1} \otimes b_{2} \otimes b_{3}\right) \\
& =0
\end{aligned}
$$

This proves the proposition.

\subsection{Tensor product of $G_{i}$-coalgebras}

Let $\left(M_{1}, \Delta_{1}\right)$ and $\left(M_{2}, \Delta_{2}\right)$ be two Lie-admissible coalgebras and $\Delta$ the composite

$$
M_{1} \otimes M_{2} \stackrel{\Delta_{1} \otimes \Delta_{2}}{\longrightarrow}\left(M_{1} \otimes M_{1}\right) \otimes\left(M_{2} \otimes M_{2}\right) \stackrel{i d_{M_{1}} \otimes \tau \otimes i d_{M_{2}}}{\longrightarrow}\left(M_{1} \otimes M_{2}\right) \otimes\left(M_{1} \otimes M_{2}\right)
$$

If $\Delta_{1}$ is a comultiplication of $G_{i}$-coalgebra, what should be the structure of $\left(M_{2}, \Delta_{2}\right)$ such that $\Delta$ is a comultiplication of $G_{i}$-coalgebra too?

Proposition 11. Let $\left(M_{1}, \Delta_{1}\right)$ be a $G_{i}$-coalgebra and $\left(M_{2}, \Delta_{2}\right)$ a $G_{i}^{!}$-coalgebra. Then $\left(M_{1} \otimes\right.$ $\left.M_{2}, \Delta\right)$ is provided with a $G_{i}$-coalgebra structure.

Proof. Using classical notations we have

$$
\tilde{A}(\Delta)(v \otimes w)=v_{1}^{1} \otimes w_{1}^{1} \otimes v_{1}^{2} \otimes w_{1}^{2} \otimes v_{2} \otimes w_{2}-v_{1} \otimes w_{1} \otimes v_{2}^{1} \otimes w_{2}^{1} \otimes v_{2}^{2} \otimes w_{2}^{2}
$$

Let $\chi:\left(M_{1} \otimes M_{2}\right)^{\otimes 3} \rightarrow M_{1}^{\otimes 3} \otimes M_{2}^{\otimes 3}$ be the isomorphism given by

$$
\chi\left(v_{1} \otimes w_{1} \otimes v_{2} \otimes w_{2} \otimes v_{3} \otimes w_{3}\right)=v_{1} \otimes v_{2} \otimes v_{3} \otimes w_{1} \otimes w_{2} \otimes w_{3}
$$

Thus we obtain, from the hypothesis on $\Delta_{2}$

$$
\chi \circ \Phi_{G_{i}}^{M_{1} \otimes M_{2}} \circ \tilde{A}(\Delta)=\Phi_{G_{i}}^{M_{1}} \circ \tilde{A}\left(\Delta_{1}\right) \otimes\left(\Delta_{2} \otimes I d\right) \circ \Delta_{2}
$$

which is zero because $\Delta_{1}$ is a $G_{i}$-comultiplication. As $\chi$ is an isomorphism, we deduce the proposition.

Remark. In [5] we have generalized this study and defined for any quadratic operad $\mathcal{P}$ a quadratic operad $\tilde{\mathcal{P}}$ so that the tensor product of a $\mathcal{P}$-algebra with a $\tilde{\mathcal{P}}$-algebra is provided with a $\mathcal{P}$-algebra structure. In the previous case we have always $\tilde{\mathcal{P}}=\mathcal{P}$ ! 


\section{Acknowledgement}

This work has been supported for the two authors by AUF project MASI 2005-2006.

\section{References}

[1] A. A. Albert. Power-associative rings. Trans. Amer. Math. Soc., 64 (1948), 552-593.

[2] V. Ginzburg and M. Kapranov. Koszul duality for operads. Duke Math J., 76 1, (1994), 203-272.

[3] M. Goze and E. Remm. Lie-admissible algebras and operads. J. Algebra, 273 (2004), 129152.

[4] M. Goze and E. Remm. A class of nonassociative algebras. Algebra Colloq., 2007 (to be published).

[5] M. Goze and E. Remm. The quadratic operad $\tilde{\mathcal{P}}$ and tensor products of algebras. Preprint, arXiv: math.RA/0606105, 2006.

[6] H. C. Myung. Lie algebras and Flexible Lie-admissible Algebras. Hadronic Press, 1982.

[7] H. C. Myung, Editor. Mathematical Studies in Lie-Admissible Algebras. Hadronic Press, Volumes I and II in 1984, Volume III in 1986.

[8] M. Markl and E. Remm. Algebras with one operation including Poisson and other Lieadmissible algebras. J. Algebra, 299 (2006), 171-189.

[9] E. Remm. Opérades Lie-admissibles. C. R. Math. Acad. Sci. Paris, 334 (2002), 1047-1050. 